\title{
Multiple nodal solutions for the Schr \" odinger-Poisson system with an asymptotically cubic term
}

\author{
Hui Guo ${ }^{1}$, Ronghua Tang ${ }^{1}$, and Tao Wang ${ }^{1}$ \\ ${ }^{1}$ Hunan University of Science and Technology
}

July 10, 2021

\begin{abstract}
This paper deals with the following Schr $\backslash$ "odinger-Poisson system \begin \{equation } \} \backslash \text { left } \backslash \{ \backslash \text { begin } \{ \text { aligned } \} \text { \&- } \backslash \text { Delta } u + u + $\backslash$ lambda $\backslash$ phi $\mathrm{u}=\mathrm{f}(\mathrm{u}) \backslash$ quad $\backslash \operatorname{mbox}\{$ in $\} \backslash \operatorname{mathbb}\{\mathrm{R}\}^{\wedge} 3, \backslash \backslash \&$ \& $\backslash$ Delta $\backslash$ phi $=\mathrm{u}^{\wedge}\{2\} \backslash$ quad $\backslash \operatorname{mbox}\{$ in $\} \backslash \operatorname{mathbb}\{\mathrm{R}\}^{\wedge} 3, \backslash$ end $\{$ aligned $\} \backslash$ right. $\backslash$ end $\{$ equation where $\$ \backslash$ lambda $>0 \$$ and $\$ \mathrm{f}(\mathrm{u}) \$$ is a nonlinear term asymptotically cubic at the infinity. Taking advantage of the Miranda theorem and deformation lemma, we combine some new analytic techniques to prove that for each positive integer $\$ \mathrm{k}, \$$ system $\backslash$ eqref\{zhaiyaofc\} admits a radial nodal solution $\$ \mathrm{U}_{-} \mathrm{k}^{\wedge}\{\backslash$ lambda\} $\$$, which has exactly $\$ \mathrm{k}+1 \$$ nodal domains and the corresponding energy is strictly increasing in $\$ \mathrm{k} \$$. Moreover, for any sequence $\$ \backslash\{\backslash$ lambda_n $\backslash\} \backslash$ to $0_{-}+\$$ as $\$ \mathrm{n} \backslash$ to $\backslash$ infty, $\$$ up to a subsequence, $\$ \mathrm{U}_{-} \mathrm{k}^{\wedge}\left\{\backslash\right.$ lambda_n\} $\$$ converges to some $\$ \mathrm{U}_{-} \mathrm{k}^{\wedge} 0 \backslash$ in $\mathrm{H}_{\mathbf{1}} \mathrm{r}^{\wedge} 1\left(\backslash \operatorname{mathbb}\{\mathrm{R}\}^{\wedge} 3\right) \$$, which is a radial nodal solution with exactly $\$ \mathrm{k}+1 \$$ nodal domains of $\backslash$ eqref $\{$ zhaiyaofc $\}$ for $\$ \backslash$ lambda=0 $\$$. These results give an affirmative answer to the open problem proposed in [Kim S, Seok J. Commun. Contemp. Math., 2012] for the Schr\"odinger-Poisson system with an asymptotically cubic term.
\end{abstract}

\section{Hosted file}

New Manuscript.pdf available at https://authorea.com/users/424852/articles/529868-multiplenodal-solutions-for-the-schr-odinger-poisson-system-with-an-asymptotically-cubic-term 